\title{
Portal thrombosis complicated by a digestive hemorrhage revealing a primary polycythemia
}

\begin{abstract}
A case report of a 30years old patient followed for portal Vein Thrombosis (PVT) complicated with upper gastrointestinal hemorrhage due to esophageal varices. JAK 2 V617F mutation was positive, therefore the diagnosis of primary polycythemia was retained. This case report showed that it important to think of different etiologies besides cirrhosis while managing patients with PVT especially diseases with pro thrombotic state.
\end{abstract}

Keywords: splanchnic vein thromboses, myeloproliferative neoplasms digestive hemorrhage, pro thrombotic state
Volume I2 Issue 2 - 202I

Chait Yassine,' Lairani Fatima Ezzahra,'
Ouaya Hassan,' Ait Errami Adil,' Samlani
Zouhour,' Krati Khadija,' Oubaha Sofia²
'Departement of Hepato-Gastro-Enterology, Mohammed VI
University Hospital Center, Morocco
'Departement of Physiology, Cadi Ayyad University, Morocco

Correspondence: Chait Yassine, Departement of HepatoGastro-Enterology, Arrazi hospital, Mohammed VI University Hospital Center, Marrakech 40000, Morocco,

Tel +212678099856, Email Yassine.chait@gmail.com

Received: December 17, 2020 | Published: April 23, 2021

\section{Introduction}

Portal vein thrombosis (PVT) is a vascular disease encountered in medical practice. It can be acute or chronic. Patients can be asymptomatic, they can present with acute symptoms when the thrombosis is acute, or present with complications due to portal hypertension at the chronic stage. ${ }^{1}$

Vaquez disease $(\mathrm{VM})$ is one of the most common myeloproliferative syndromes with an incidence in Europe of around 0.4 to 2.6 per 100,000 populations. VM is known to moderately increase the risk of venous and / or arterial thrombotic episodes. However, portal thromboses are rarely reported in the literature. It has been suggested that Myeloproliferative neoplasms MPNs presenting with splanchnic vein thromboses SVT are biologically different entities because they usually show less altered blood counts, low JAK2V617F allele burden load, and a low rate of disease progression. ${ }^{2}$

The present study was planned to point at the importance of thinking of MPNs while managing SVT, as well as raise knowledge about its impact on the underlying condition.

\section{Observation}

Thirty-years-old patient, followed for 2 years for a portal vein thrombosis without identified etiology, put on anticoagulant treatment with anti-vitamin K (AVK). During a hospitalization for upper gastrointestinal hemorrhage (hematemesis and melena). Clinical examination revealed splenomegaly.

The results showed: $\mathrm{Hb}=8.9 \mathrm{~g} / \mathrm{dl}$, Hematocrit $=27.3 \%, \mathrm{VGM}=75.4$ fl, Platelets $=12190 / \mathrm{mm}^{3}$, Leukocytes $=22690 \mathrm{~g} / \mathrm{l}$, PT $=48 \%$, aPTT $=1.3$, a ferritin $=4 \mathrm{ng} / \mathrm{ml}$, normal kidney function and liver enzymes. The esogastroduodenal fibroscopy objectified grade III esophageal varices. Ultrasound revealed the presence of splenomegaly. The search for the JAK 2 V617F mutation returned positive at $29 \%$.

The patient underwent ligation of the esophageal varices. Hydroxyurea cytoreductive therapy was instituted, as well as the resumption of anticoagulation by LMWH (low-molecular-weight heparin) and relay by antivitamin $\boldsymbol{k}$. The evolution was marked by a decrease in stomach pain, a reduction in the frequency of hemorrhagic episodes, a normalization of the hemogram and a decrease in the size of the splenomegaly.

\section{Discussion}

Splanchnic vein thromboses (SVTs), including portal vein thrombosis (PVT), are severe vascular events. ${ }^{3}$ The pathogenesis of SVTs is mostly dependent on the presence of systemic prothrombotic conditions such as neoplasms (MPNs). Indeed, $30 \%$ to $40 \%$ of budd chiari syndrome BCS and PVT are due to MPNs. ${ }^{4-6}$

Thrombosis of portal vein is a result of local and systemic factors. ${ }^{1}$ When the symptoms occur sixty days before presentation, PVT is therefore considered acute, it presents with acute symptoms such as fever, abdominal pain and vomiting. ${ }^{7}$ When PVT reaches a chronic stage, it is associated with formation of collateral veins and is therefore called "portal cavernoma" (Figures 1-5). ${ }^{8}$ The diagnosis of PVT involves Doppler ultrasonography, MRI, CT that are considered non-invasive methods, they have replaced invasive tests of portal vein. ${ }^{9}$

The first step in managing portal thrombosis is to determine if it is acute or chronic, and if there is liver cirrhosis (Figure 5). Several studies in the literature report the association of thrombotic accidents like PVT and conditions such as myeloproliferative disorders, it is therefore important to follow these patients adequately (Figure 6). ${ }^{11}$

There are numerous cases of PVT diagnosed as "idiopathic", that were in reality a result of prothrombotic conditions, such as Polycythemia vera (PV). ${ }^{11}$ Association between MPN and SVT just like our case, is a unique presentation due to particular disease features: young age $(<45$ years old, mostly polycythemia vera PV with a low JAK2V617F-mutant allele burden). ${ }^{12}$

Vaquez disease is a primitive polycthemia, it is secondary to an increased number of red cells. ${ }^{13}$ In PV, there are more arterial thrombosis than veinous thrombosis, the risk is higher in early stages. ${ }^{14}$ Risk factors of thrombosis include age over sixty years, thrombosis history, elevated hematocrit, platelet count and leukocytes (Figure 7). ${ }^{14}$ 

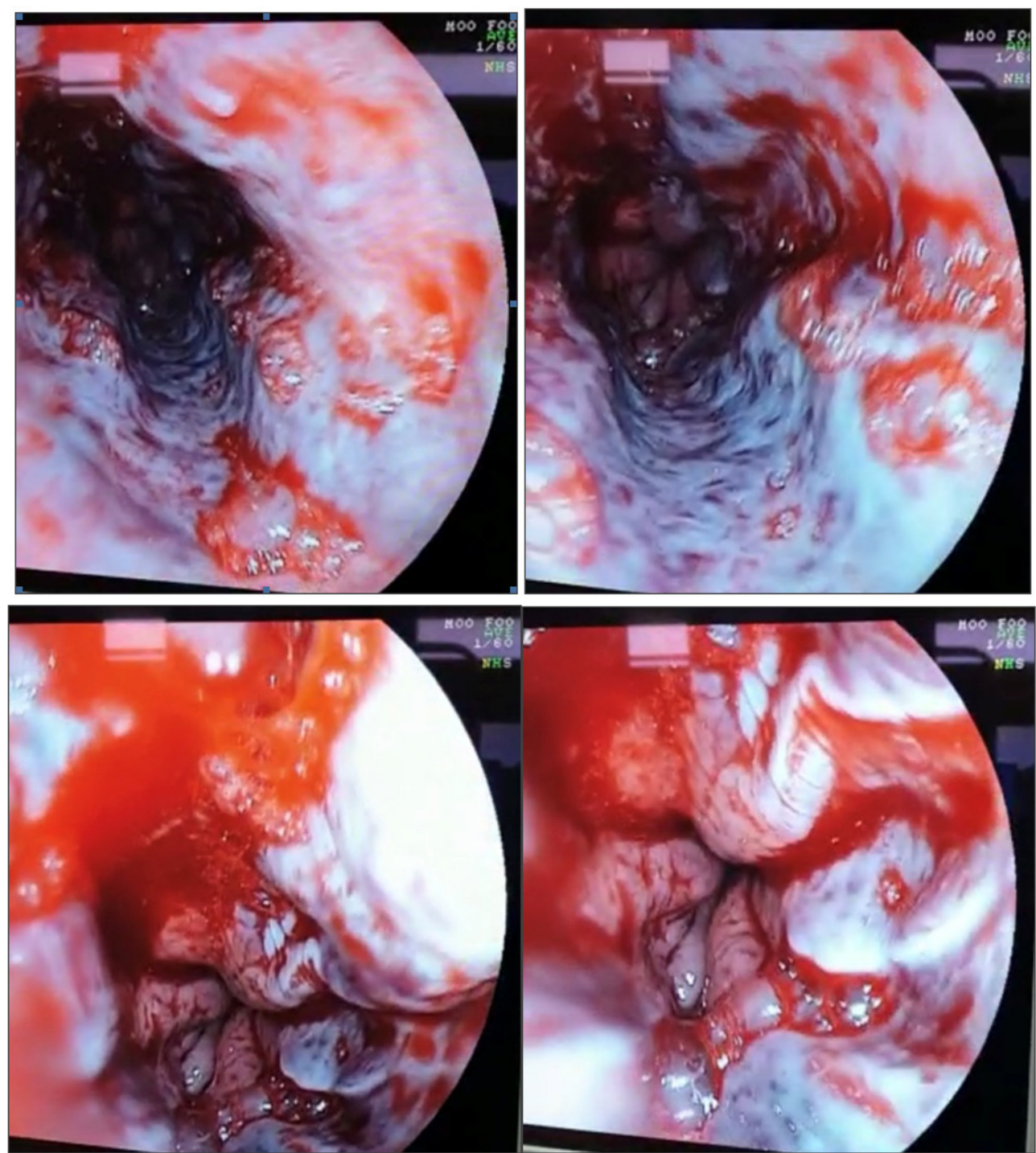

Figure I Endoscopic aspect of grade III esophageal varices.

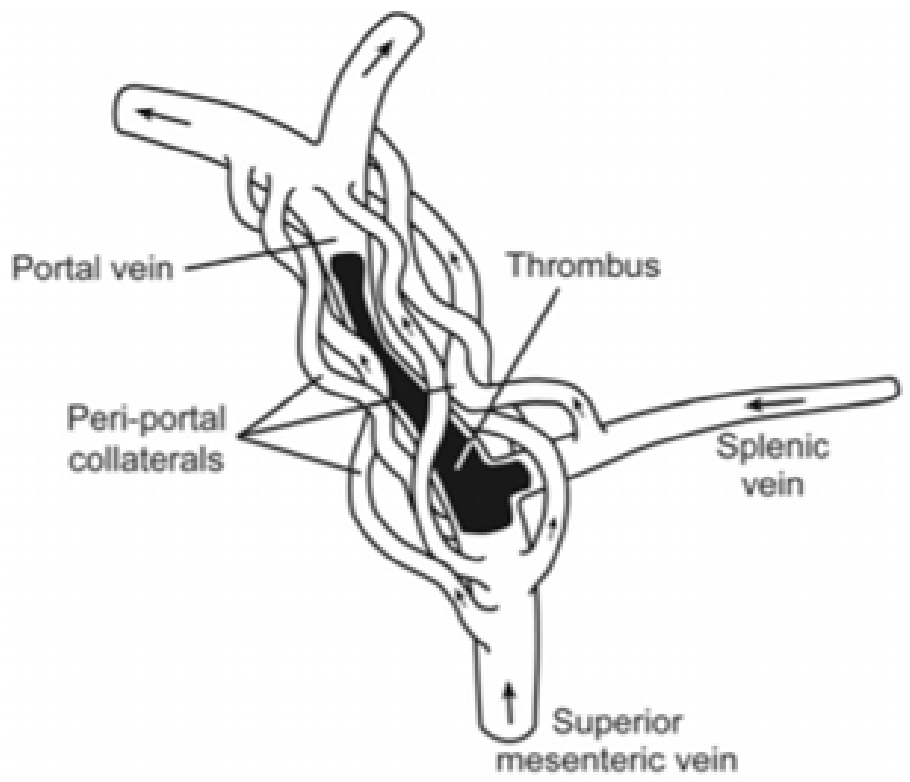

Figure 2 Chronic splanchnic vein thrombosis. ${ }^{8}$

Citation: Yassine C, Ezzahra LF, Hassan O, et al. Portal thrombosis complicated by a digestive hemorrhage revealing a primary polycythemia. Gastroenterol Hepatol Open Access. 2021;12(2):47-52. DOI: 10.15406/ghoa.202I.I2.00456 

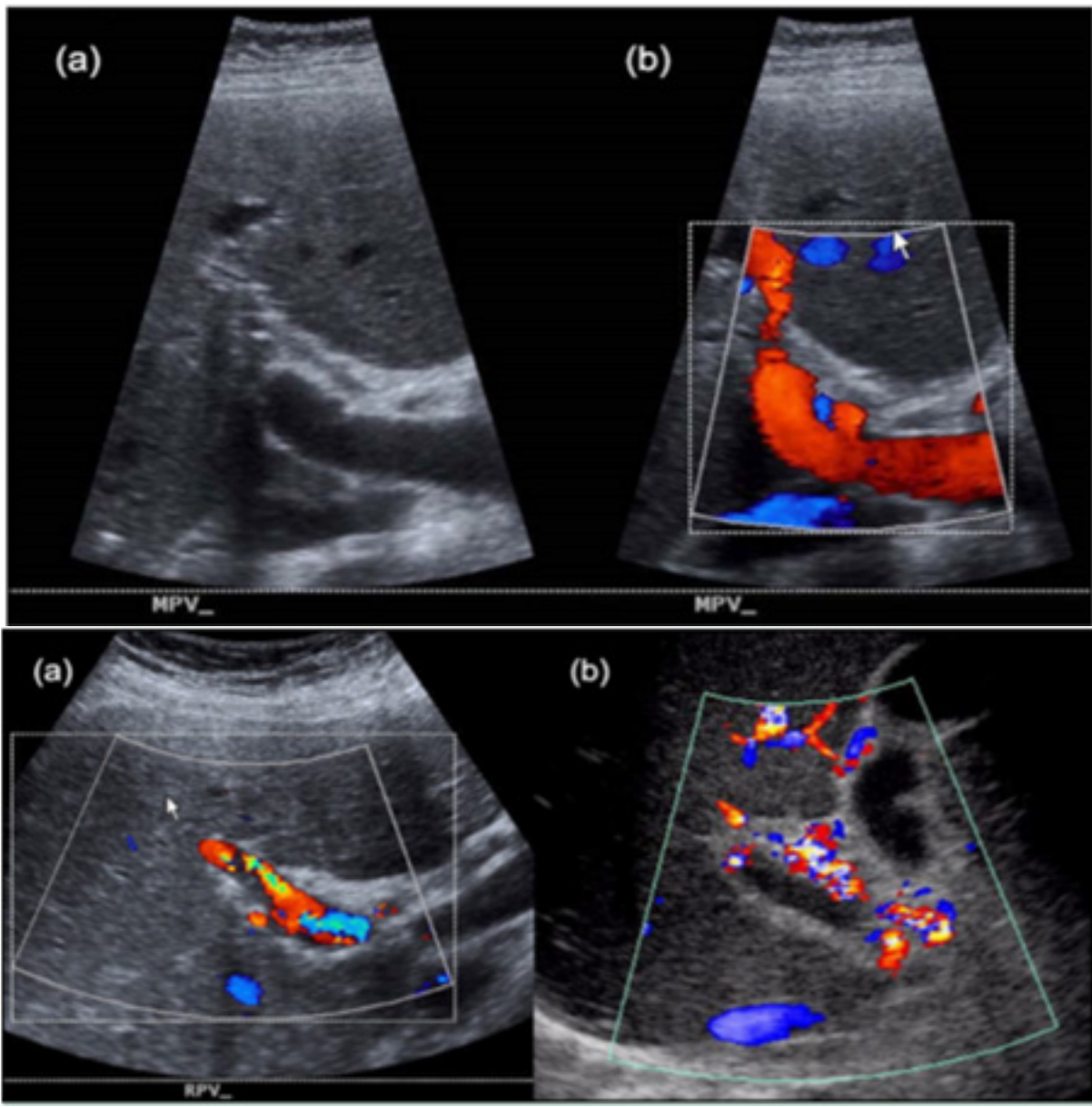

Figure 3 Ultrasound of the abdomen showing normal portal vein and portal vein thrombosis. ${ }^{7}, 10$
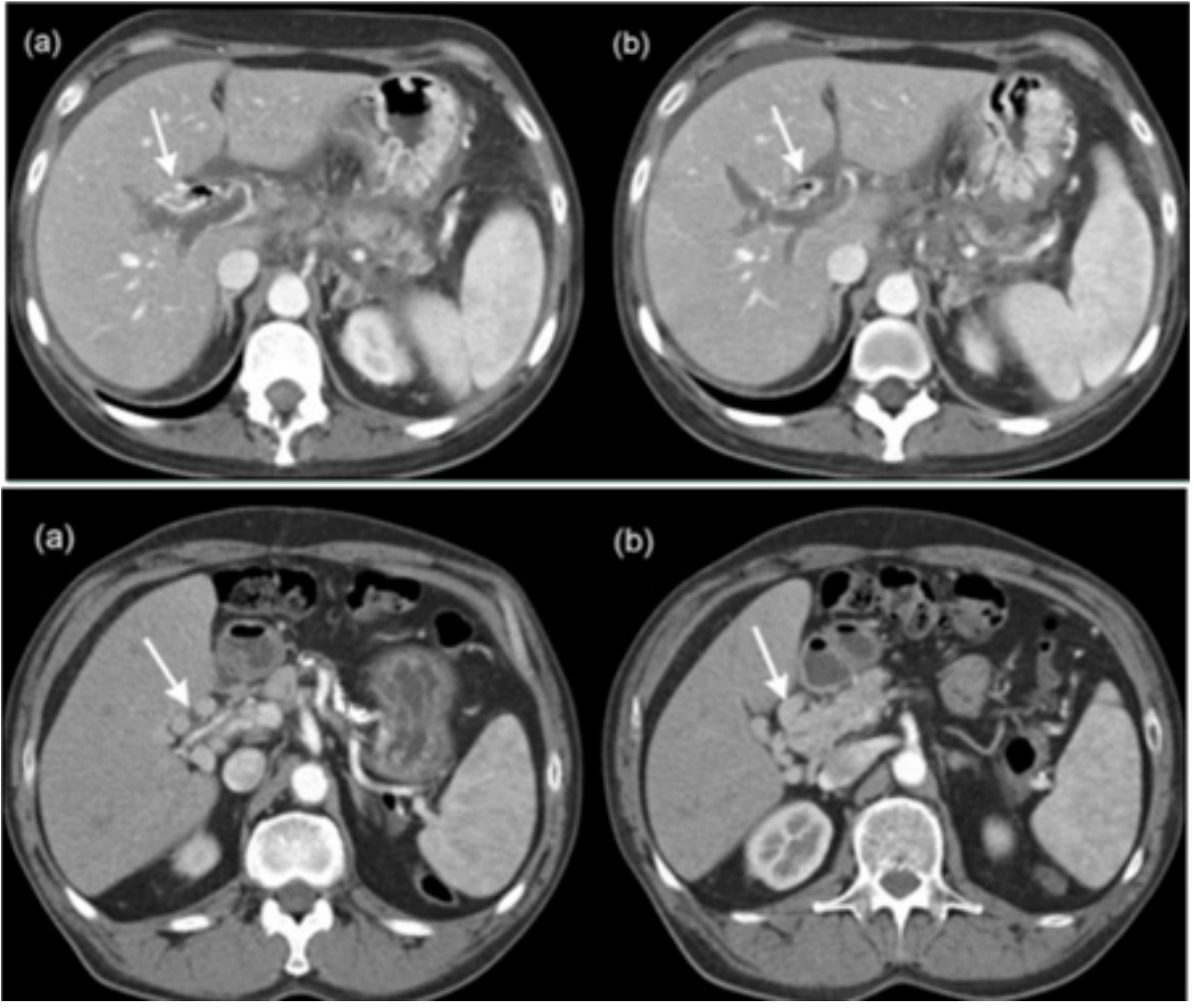

Figure 4A, B Abdomen tomography showing acute portal thrombosis and chronic portal thrombosis with portal cavernoma. ${ }^{1,9}$

Citation: Yassine C, Ezzahra LF, Hassan O, et al. Portal thrombosis complicated by a digestive hemorrhage revealing a primary polycythemia. Gastroenterol Hepatol Open Access. 2021;12(2):47-52. DOI: 10.15406/ghoa.202I.I2.00456 


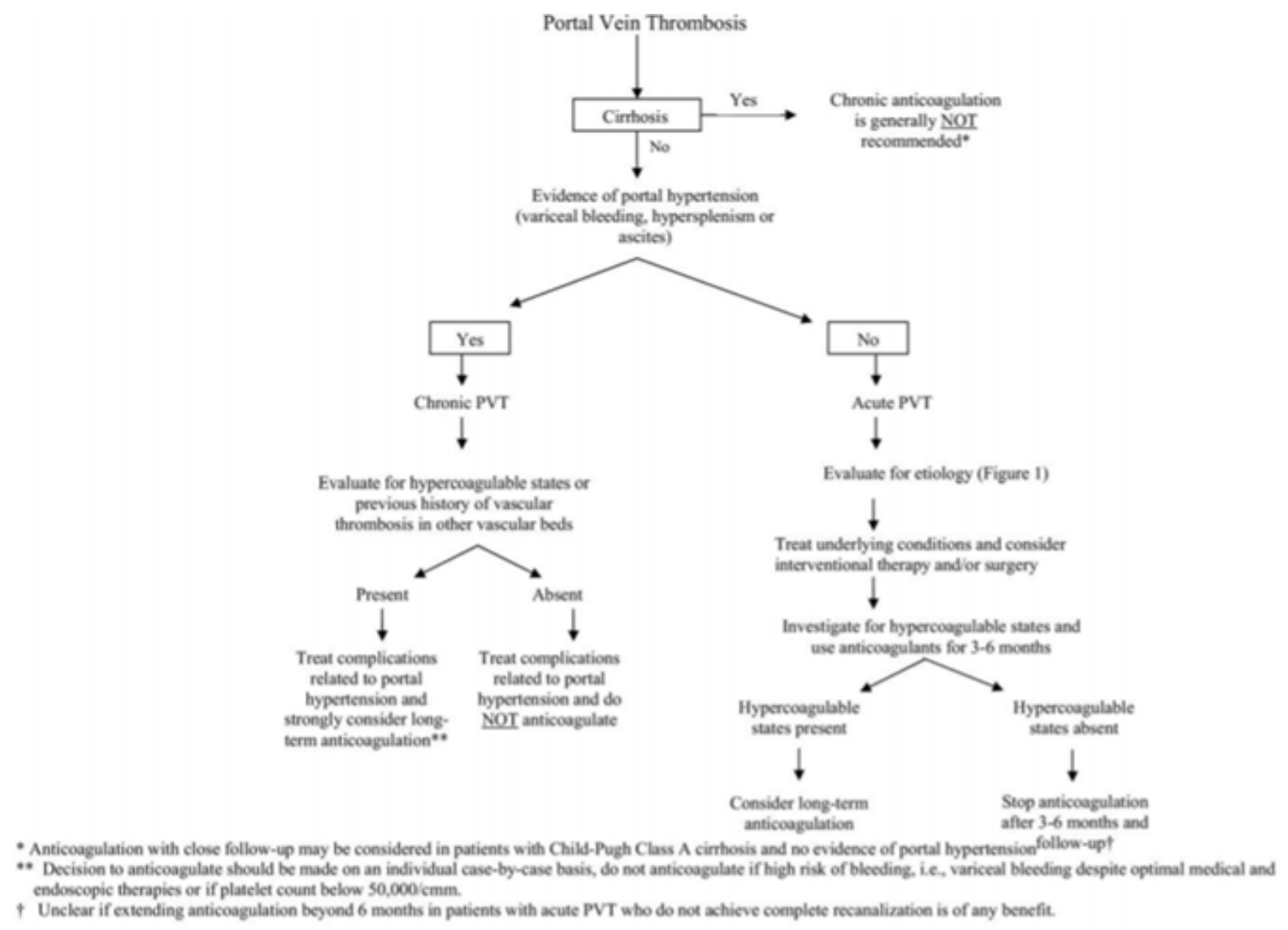

Figure 5 A proposed approach of portal thrombosis'

\section{Etiology}

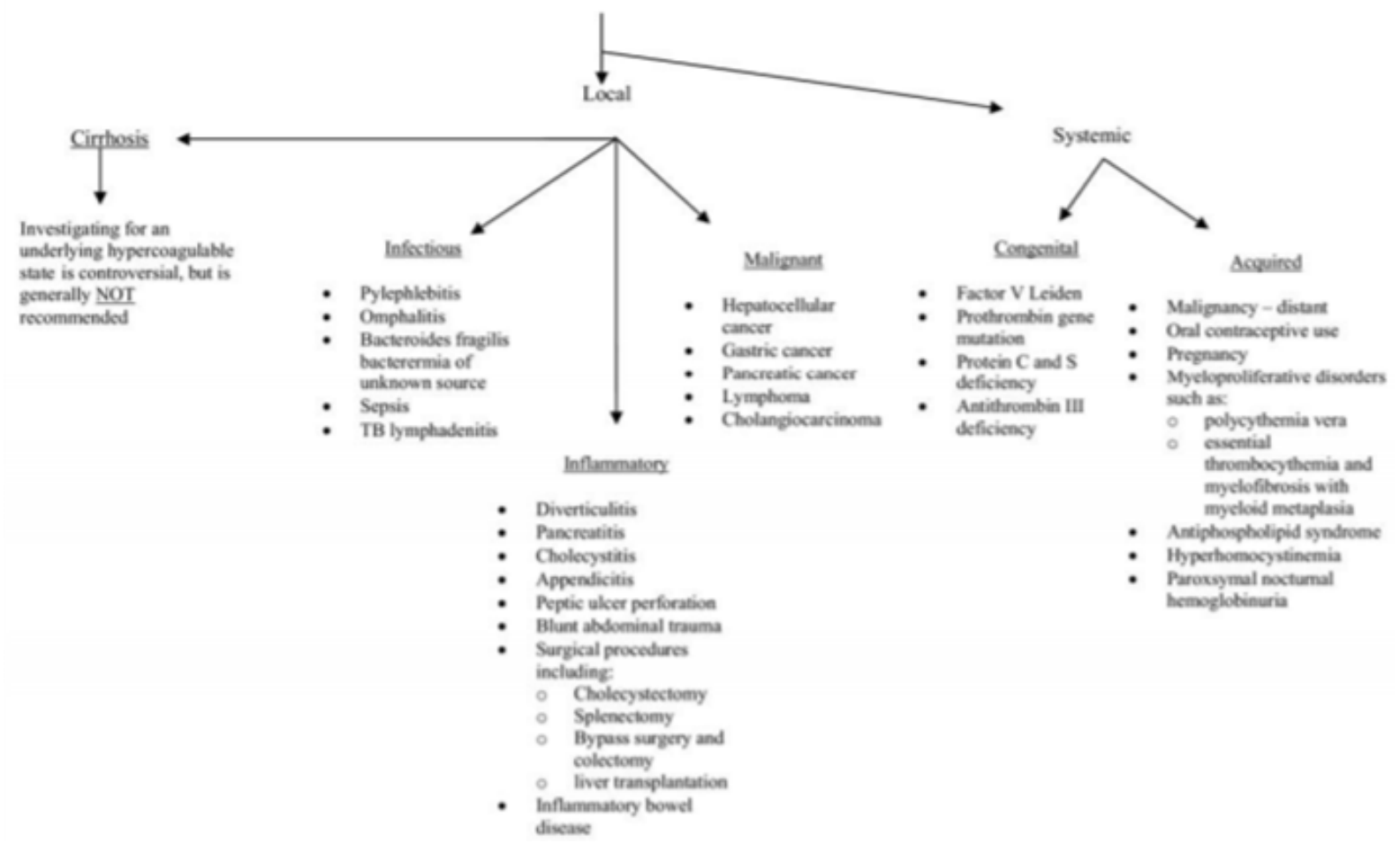

Figure 6 Etiology of portal thrombosis' 


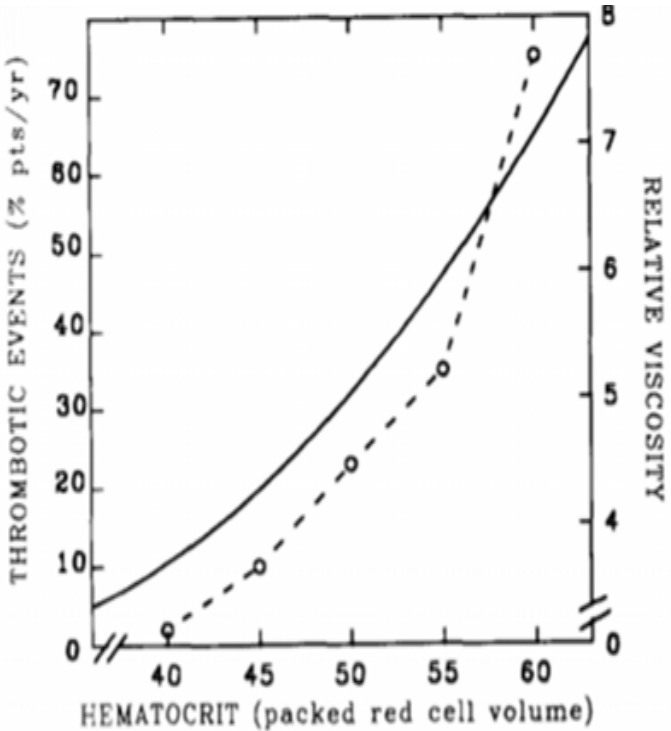

Figure 7 Incidence of thrombosis in PV patients depending on blood viscosity. ${ }^{15}$

Janus kinase 2 mutation, was found in up to thirty per cent of patients with PVT. ${ }^{16}$ Its incidence is ninety five per cent approximately in patients with PV. ${ }^{1}$ Debureaux and al. developed a stratification allowing identifying high risk patients that would present thrombotic events. The patients harboring 1 or both of the molecular risk factors: JAK2V617F allele burden $50 \%$ or presence of chromatin/ spliceosome/TP53 mutation. ${ }^{12}$ The presence of SVT in PV was in many studies considered as a prognostic factor, in a matter of fact, Alvarez and al. described an increase in progression to myelofibrosis in patients presenting the association SVT-PV. ${ }^{2}$

De Stefano et al. ${ }^{17}$ have reported in another study an incidence of severe bleeding of $2.1 \%$ per year in patients with MPNs-SVT, this can be explained by the use of anticoagulant therapy and the presence of esophageal varices EV (Figure 8).

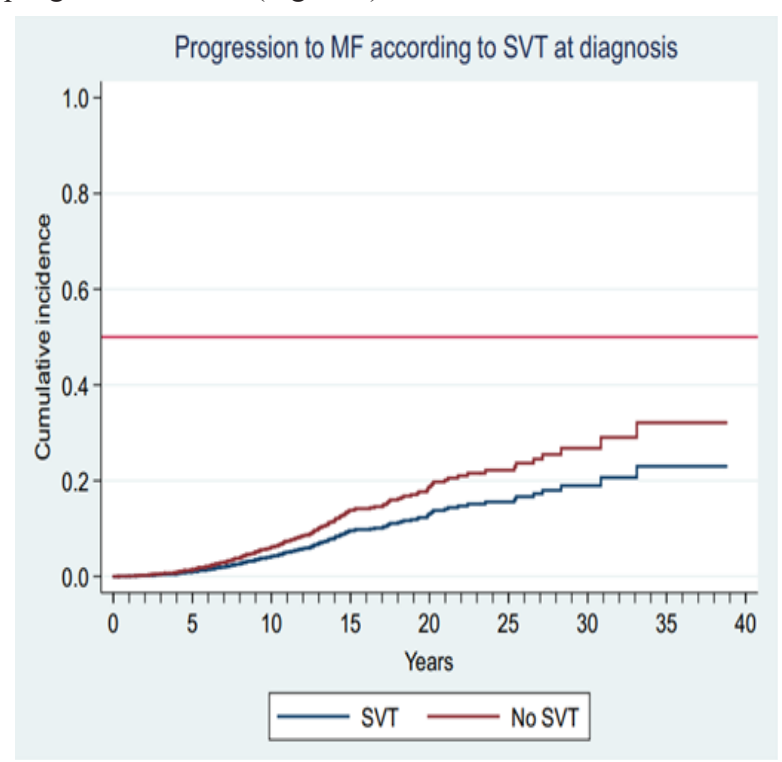

Figure 8 Estimated probability of progression to myelofibrosis (MF) adjusted by age and sex in polycythemia vera and essential thrombocythemia patients with splanchnic vein thrombosis (SVT) at diagnosis (blue line) or not (red line) p not significant. ${ }^{2}$
The choice of treatment must take into account two objectives: ${ }^{13}$

- Reduce blood viscosity in order to avoid complications vascular

- Limit the frequency of haematological changes: myelofibrosis and acute leukemia

The treatment indications are adapted to the risk. Patients with a low risk (age inferior to sixty years and no history of thrombosis) are treated with low dose bleeds and aspirin, while patients with a higher risk (age over sixty years and / or history of thrombosis) should receive 'hydroxyurea. ${ }^{13}$ Peg-interferon alpha-2a can be used, mainly in young people and seems to give results similar to hydroxyurea. Cytoreductive therapy significantly reduces the incidence of cardiovascular events. ${ }^{13}$ Management of MPN-SVT is challenging given their composite vascular risk that includes a high risk of both thrombotic recurrence and major bleeding, especially from EV. Though there is a general agreement in maintaining long-term anticoagulation, valuing the underlying hematological disorder as a permanent risk factor recurrent thromboses - either SVT or not - are reported in a nontrivial proportion of MPN-SVT. ${ }^{17}$ Moreover, the SVT impact on MPNs patients mortality is still controversial. ${ }^{17}$ The aim of the present study was to improve the knowledge of MPN-SVT, it also showed that SVT can be the first symptom revealing an underlying MPN such as PV.

\section{Conclusion}

Portal thrombosis is a rarely revealing presentation of primary polycythemia. Early diagnosis improves the prognosis by reducing morbidity and mortality. For this reason, it is recommended to think of MPNs especially PV as an etiology while managing PV.

\section{Acknowledgments}

None.

\section{Conflicts of interest}

Authors declare that we have no conflicting interests.

\section{Funding}

The authors did not receive any grant for this research study.

\section{References}

1. Parikh S, Shah R, Kapoor P. Portal vein thrombosis. Am J Med. 2010;123(2):111-119.

2. Alvarez-Larrán A, Pereira A, Magaz M, et al. Natural history of polycythemia vera and essential thrombocythemia presenting with splanchnic vein thrombosis. Ann Hematol. 2020;99(4):791-798.

3. Plessier A, Rautou P-E, Valla D-C. Management of hepatic vascular diseases. J Hepatol. 2012;56:S25-S38.

4. Liver EAFTSOT. EASL Clinical Practice Guidelines: Vascular diseases of the liver. J Hepatol. 2016;64(1):179-202.

5. Stein BL, Martin K. From Budd-Chiari syndrome to acquired von Willebrand syndrome: thrombosis and bleeding complications in the myeloproliferative neoplasms. Hematology. 2019;2019(1):397-406.

6. Smalberg JH, Arends LR, Valla DC, et al. Myeloproliferative neoplasms in Budd-Chiari syndrome and portal vein thrombosis: a meta-analysis. Blood J Am Soc Hematol. 2012;120(25):4921-4928.

7. Gertsch P, Matthews J, Lerut J, et al. Acute thrombosis of the splanchnic veins. Arch Surg. 1993;128(3):341-345. 
8. Cohen J, Edelman RR, Chopra S. Portal vein thrombosis: a review. Am J Med. 1992;92(2):173-182.

9. Haddad MC, Clark DC, Sharif HS, et al. MR, CT, and ultrasonography of splanchnic venous thrombosis. Gastrointest Radiol. 1992;17(1):34-40.

10. Ueno N, Sasaki A, Tomiyama T, et al. Color Doppler ultrasonography in the diagnosis of cavernous transformation of the portal vein. $J$ Clin Ultrasound. 1997;25(5):227-233.

11. Chait Y, Condat B, Cazals-Hatem D, et al. Relevance of the criteria commonly used to diagnose myeloproliferative disorder in patients with splanchnic vein thrombosis. Br J Haematol. 2005;129(4):553-560.

12. Debureaux PE, Cassinat B, Soret-Dulphy J, et al. Molecular profiling and risk classification of patients with myeloproliferative neoplasms and splanchnic vein thromboses. Blood Adv. 2020;4(15):3708-3715.
13. Tulliez M. Polyglobulie de Vaquez. Rev Fr Lab. 1997;1997(296):21-26.

14. Landolfi R, Rocca B, Patrono C. Bleeding and thrombosis in myeloproliferative disorders: mechanisms and treatment. Crit Rev Oncol Hematol. 1995;20(3):203-222.

15. Pearson TC, Wetherley-Mein G. Vascular occlusive episodes and venous haematocrit in primary proliferative polycythaemia. Lancet. 1978;312(8102):1219-1222.

16. Kiladjian JJ, Cervantes F, Leebeek FW, et al. The impact of JAK2 and MPL mutations on diagnosis and prognosis of splanchnic vein thrombosis: a report on 241 cases. Blood. 2008;111(10):4922-4929.

17. De Stefano V, Vannucchi AM, Ruggeri M, et al. Splanchnic vein thrombosis in myeloproliferative neoplasms: risk factors for recurrences in a cohort of 181 patients. Blood Cancer J. 2016;6(11):e493-e493. 